\title{
Progressive Encephalomyelitis with Rigidity and Myoclonus (PERM)-like Symptoms Associated with Anti-ganglionic Acetylcholine Receptor Antibodies
}

\author{
Yuki Kitazaki ${ }^{1,2}$, Masamichi Ikawa ${ }^{2,3}$, Toru Kishitani ${ }^{1,2}$, Tomoko Kamisawa ${ }^{1,2}$, \\ Shunya Nakane ${ }^{4}$, Yasunari Nakamoto ${ }^{2}$ and Tadanori Hamano ${ }^{2,5}$
}

\begin{abstract}
:
This report describes a 59-year-old woman who presented with progressive encephalomyelitis with rigidity and myoclonus (PERM)-like symptoms and severe dysautonomia, including orthostatic hypotension, sinus bradycardia, dysuria, and prolonged constipation. Her neurological symptoms improved after immunotherapy, but the dysautonomia persisted. Anti-ganglionic acetylcholine receptor (gAChR) $\alpha 3$ subunit antibodies, which are frequently identified in patients with autoimmune autonomic ganglionopathy, were detected in the pretreatment serum. The central distribution of the nicotinic acetylcholine receptors, a target of anti-gAChR antibodies, and immunotherapeutic efficacy observed in this case indicate that anti-gAChR $\alpha 3$ subunit antibodies are associated with the PERM-like features accompanied by autonomic manifestations.
\end{abstract}

Key words: progressive encephalomyelitis with rigidity and myoclonus, anti-ganglionic acetylcholine receptor antibodies, autoimmune encephalitis, autonomic failure, autoimmune autonomic ganglionopathy

(Intern Med 60: 2307-2313, 2021)

(DOI: 10.2169/internalmedicine.6419-20)

\section{Introduction}

Progressive encephalomyelitis with rigidity and myoclonus (PERM), an autoimmune encephalitis, is a variant of stiff-person syndrome with autonomic failure $(1,2)$. Autoantibodies to glutamic acid decarboxylase (GAD), glycine receptors (GlyR), N-methyl-D-aspartate receptors (NMDAR), and dipeptidyl-peptidase-like protein 6 (DPPX) have been reported to be associated with PERM (3-6).

We herein report a case that showed PERM-like symptoms with persistent dysautonomia and harbored antiganglionic acetylcholine receptor (gAChR) $\alpha 3$ subunit antibodies. The nicotinic acetylcholine receptors (nAChRs), which are a target of anti-gAChR antibodies, are widely distributed both in the central nervous system (CNS) and pe- ripheral autonomic nerves (7). The distribution of nAChRs advocates the relationship between positive anti-gAChR $\alpha 3$ subunit antibodies and the presentation of PERM-like symptoms with persistent dysautonomia in the patient.

\section{Case Report}

A 59-year-old Japanese woman developed a problem with writing due to tremors in her fingers on the first day of symptom onset (day 1). She had a past medical history of congenital hip dysplasia after bilateral hip replacement, had no particular family history, and took no medications. She presented with hearing loss and enteritis-like symptoms such as fever, vomiting, and diarrhea on day 5. In addition, since she suffered from dysphagia and had difficulty eating, she was admitted to the previous hospital on day 10. Walking

\footnotetext{
${ }^{1}$ Department of Neurology, Fukui-ken Saiseikai Hospital, Japan, ${ }^{2}$ Second Department of Internal Medicine, Faculty of Medical Sciences, University of Fukui, Japan, ${ }^{3}$ Department of Advanced Medicine for Community Healthcare, Faculty of Medical Sciences, University of Fukui, Japan, ${ }^{4}$ Department of Neurology, Graduate School of Medical Sciences, Kumamoto University, Japan and ${ }^{5}$ Department of Aging and Dementia, Faculty of Medical Sciences, University of Fukui, Japan

Received: October 1, 2020; Accepted: December 26, 2020; Advance Publication by J-STAGE: February 15, 2021

Correspondence to Dr. Masamichi Ikawa, iqw@u-fukui.ac.jp
} 

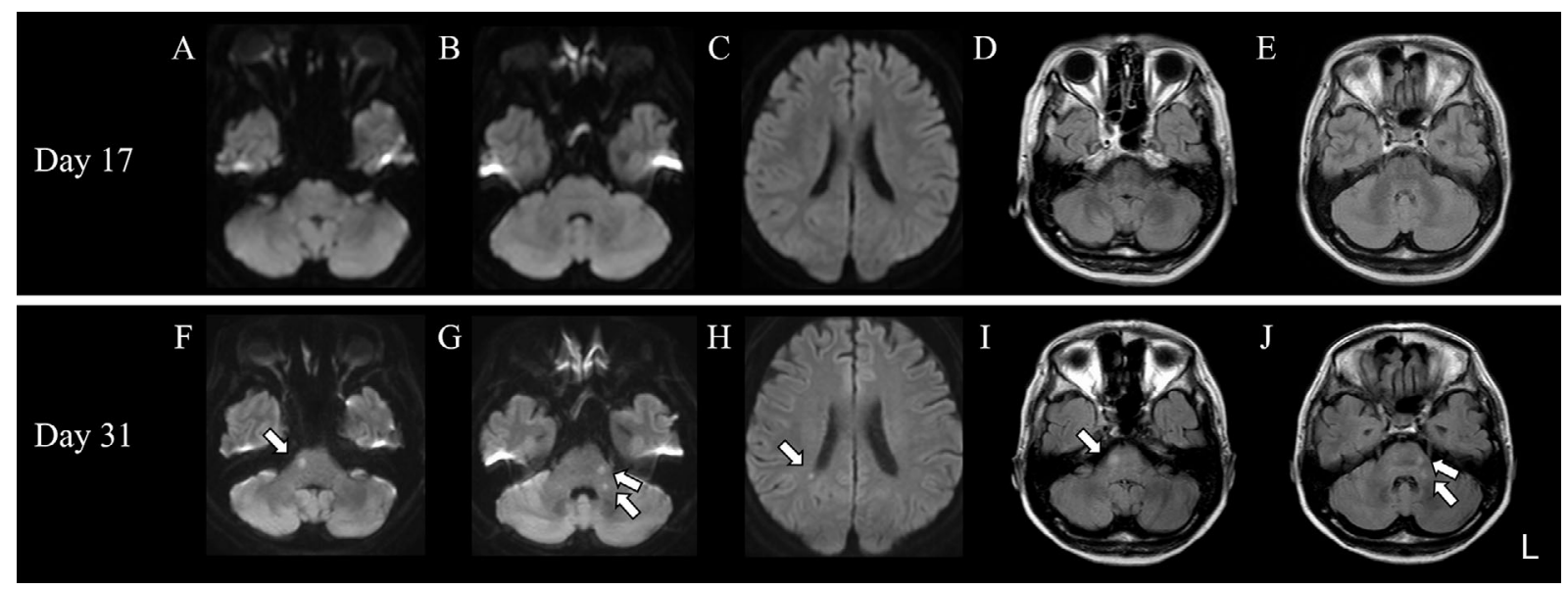

Figure 1. Brain MRI on day 17 and on day 31. (A-C) Diffusion-weighted imaging (DWI) (1.5 Tesla; TR 2,930.2 ms, TE $75.1 \mathrm{~ms}$ ) and (D, E) fluid-attenuated inversion recovery (FLAIR) images without contrast (1.5 Tesla; TR 10,000 ms, TE $120.0 \mathrm{~ms})$ showed normal findings on day 17. (F-H) DWI (3.0 Tesla; TR 5,000.0 ms, TE $60.1 \mathrm{~ms}$ ) revealed scattered lesions in the cerebral white matter and brainstem (arrows). (I, J) FLAIR images (3.0 Tesla; TR 11,000 ms, TE $125.0 \mathrm{~ms}$ ) revealed scattered lesions in the brainstem (arrows) on day 31.

difficulties related to stiffness and bradykinesia appeared on day 12. At the time, she presented with disorientation, nystagmus, a limitation of abduction of the left eye, rigidity in the right limb, dysuria, and paralysis of both upper limbs. Brain magnetic resonance imaging (MRI) showed normal findings on day 14. A cerebrospinal fluid (CSF) analysis revealed an elevated cell count (58 cells $/ \mu \mathrm{L})$, high protein $(127.0 \mathrm{mg} / \mathrm{dL})$, and normal levels of glucose $(82 \mathrm{mg} / \mathrm{dL})$ on day 15 . She was treated with meropenem (3 g/day), acyclovir (1,500 mg/day), and dexamethasone (19.8 mg/day) as she was suspected to have encephalitis. On day 17, she was transferred to our hospital with a progressively impaired consciousness.

On admission, she had a mild fever (axillary body temperature, $37.5^{\circ} \mathrm{C}$ ) and occasionally showed sinus bradycardia (minimum heart rate: 34 beats per minute). Her thyroid gland was not enlarged. In her neurological examination, the Glasgow Coma Scale was E2V1M4. She presented with ocular flutter, muscle stiffness and rigidity of limbs, myoclonus around the mouth and abdomen, dysuria, and brisk tendon reflexes with no pathological reflex. One hour after hospitalization, she developed persistent generalized tonicclonic seizures, resulting in emergent intubation and mechanical ventilation in the intensive care unit.

A routine blood analysis showed a normal leukocyte count, thyroid function, creatine phosphokinase, and Creactive protein levels. Serological tests for systemic infection including procalcitonin, $(1 \rightarrow 3)-\beta-D$-glucan, human immunodeficiency virus, cytomegalovirus (CMV), and EpsteinBarr virus (EBV) were negative. Serological tests for autoimmune diseases including anti-nuclear antibodies, antiaquaporin 4 antibodies, anti-SS-A/-B antibodies, and antiglycolipid (GM1, GQ1b) antibodies were negative. Antithyroid peroxidase (TPO) antibodies and anti-thyroglobulin (Tg) antibodies were both positive when assessed by electro- chemiluminescence immunoassay at titers of 1,777.5 IU/mL and $71.8 \mathrm{IU} / \mathrm{mL}$, respectively. CSF analysis revealed elevated cell count ( 73 cells/ $\mu \mathrm{L}, 90 \%$ monocytes), high protein (112.4 mg/dL), and normal levels of glucose $(92 \mathrm{mg} / \mathrm{dL})$ with negative results for infectious studies for CMV, EBV, herpes simplex virus, and bacterial cultures. CSF-IgG index was elevated (0.91). The test results for myelin basic protein and oligoclonal bands were both negative. Screening for PERM- or autoimmune encephalitis-associated antibodies including GAD, GlyR, NMDAR, DPPX, alpha-amino-3hydroxy-5-methyl-4-isoxazolepropionic acid receptor (AMPAR), leucine-rich glioma-inactivated 1 (LGI1), gammaaminobutyric acid $B$ receptor $\left(\mathrm{GABA}_{\mathrm{B}} \mathrm{R}\right)$, contactinassociated protein-like 2 (Caspr2), metabotropic glutamate receptor (mGluR) 1 and 5 , and $\mathrm{NH}_{2}$-terminal of $\alpha$-enolase (NAE) yielded negative results. Contrast-enhanced brain MRI revealed normal findings on diffusion-weighted images (DWI) and fluid-attenuated inversion recovery (FLAIR) images, except for a small meningeal enhancement of the right parietal lobe on day 17 (Fig. 1A-E). The postictal electroencephalogram (EEG) exhibited generalized slow waves without epileptic discharge. Truncal computed tomography (CT) showed no evidence of cancer. A nerve conduction study (NCS) demonstrated normal compound muscle action potential (CMAP) amplitudes and nerve conduction velocities (NCV), but F-wave disappearance was observed in the right median and ulnar nerves.

The patient was clinically suspected to have autoimmune encephalitis including PERM and was treated with parenteral methylprednisolone (1 g/day for 3 days, 2 cycles) and intravenous immunoglobulin $(0.4 \mathrm{mg} / \mathrm{kg} / \mathrm{day}$ for 5 days), followed by oral corticosteroid (40 mg/day). Levetiracetam $(1,000 \mathrm{mg} /$ day $)$ was administered because of persistent generalized tonic-clonic seizures and myoclonus on day 18. The patient's neurological symptoms improved after im- 

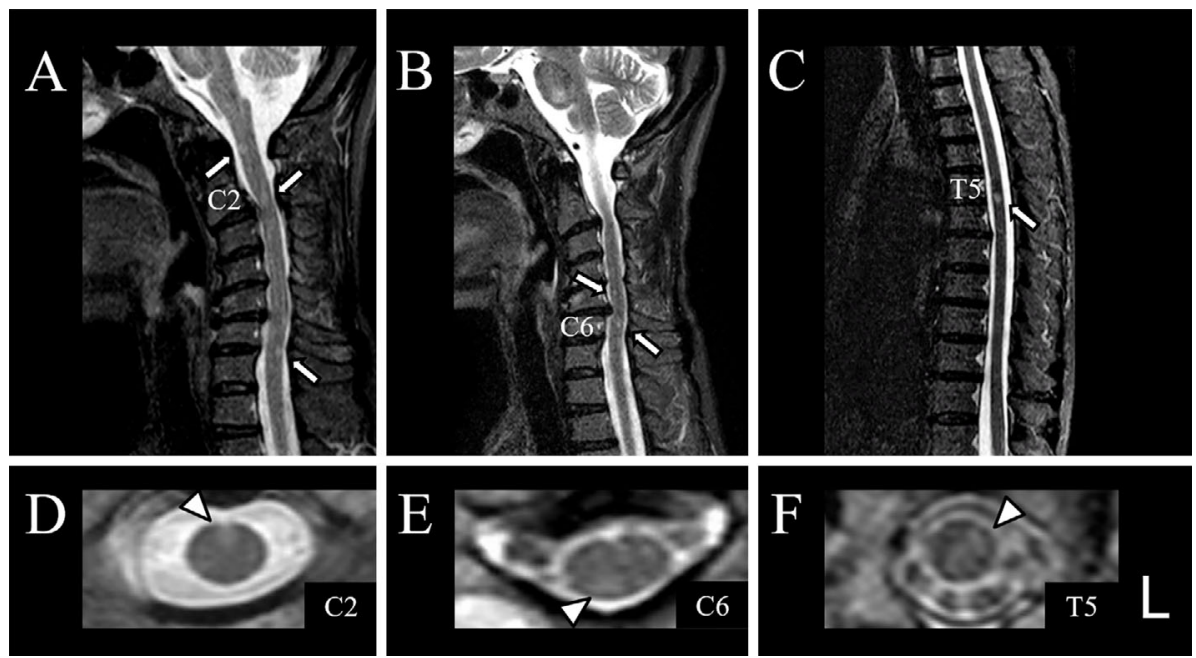

Figure 2. Spine MRI on day 32. (A-C) Sagittal spine T2-weighted short T1 inversion recovery (STIR) images (1.5 Tesla; TR 2,500.0 ms, TE $80.0 \mathrm{~ms}$ ) revealed scattered spotty lesions in the cervical and thoracic spinal cords (arrows). (D-F) Axial spine T2-weighted STIR images revealed noncentric and scattered hyperintensity lesions in the cervical and thoracic spinal cords (arrowheads).

munotherapy, and she was able to breathe spontaneously on day 23. After extubation on day 27, she became ambulatory with a cane, albeit with rigidity and bradykinesia. There was no suggestion of hyperekplexia. Brain MRI without contrast depicted scattered lesions on DWI and FLAIR images in the cerebral white matter and brainstem on day 31 (Fig. 1F-J). Spine MRI without contrast revealed scattered lesions on $\mathrm{T}$ 2-weighted short T1 inversion recovery images in the cervical and thoracic cords on day 32 (Fig. 2). Based on the clinical symptoms and features, such as encephalitis with rigidity and myoclonus, and MRI findings showing scattered lesions in brain and spine, the patient was diagnosed with autoimmune encephalomyelitis with PERM-like symptoms (4). Despite the neurological recovery, there was persistent severe autonomic failure characterized by orthostatic hypotension (systolic blood pressure dropped to $70 \mathrm{mmHg}$ without compensatory tachycardia during the Schellong test), sinus bradycardia (minimum heart rate: 40 beats per minute), dysuria with bladder retention (post-voiding residual urine volume $>150 \mathrm{~mL}$ ), and prolonged constipation was also observed. Hence, we suspected the involvement of autoantibodies associated with autonomic neuropathy. Immunological studies of the pre-treatment serum (collected on day 17) revealed positive anti-gAChR $\alpha 3$ subunit antibodies [1.18 antibody index (A.I.); normal, <1] and negative $\beta 4$ subunit antibodies (0.568 A.I.) as assessed by luciferase immune-precipitation systems (LIPS) (8). In the serum collected after continuous immunotherapy (on day 27), a negative conversion of anti-gAChR $\alpha 3$ subunit antibodies was confirmed (0.38 A.I.), consistent with the improvement in her neurological symptoms and brain-spine MRI abnormalities. Autonomic failure gradually improved with 3 months of continuous immunotherapy and recovered completely (Fig. 3). Subsequently, there was no recurrence for 2 years, even without post-immunotherapy treatment. The final diag- nosis was PERM-like autoimmune encephalomyelitis associated with anti-gAChR antibodies.

\section{Discussion}

We herein report a case of PERM-like symptoms and persistent autonomic failure with the presence of anti-gAChR $\alpha$ 3 subunit antibodies. The clinical findings in our case were characterized by ocular flutter, rigidity of limbs, myoclonus, and autonomic failure. These symptoms were consistent with PERM features, and the characteristic ensemble of symptoms can provide a clinical diagnosis for PERM $(4,9)$ (Table 1). Although rigidity, stiffness, and myoclonus are frequently observed on the surface electromyogram in patients with PERM $(6,10,11)$, we did not evaluate our case for these features because of her emergent intubation and dramatic symptom improvement after immunotherapy. Stiffperson syndrome and neuroleptic malignant syndrome are common differential diagnoses of PERM (12-14). The present case showed symptoms of brainstem involvement such as ocular flutter, hearing loss, and dysphagia, which were inconsistent with stiff-person syndrome $(9,15)$. Besides, the case had normal creatine phosphokinase values and no administration of antipsychotics suggestive of neuroleptic malignant syndrome (16). Therefore, we excluded both diseases and diagnosed this case to have PERM-like autoimmune encephalomyelitis.

The present case showed positive results of anti-gAChR $\alpha$ 3 subunit antibodies and presented CNS involvement (i.e., PERM-like symptoms) with severe dysautonomia. Our case's autonomic symptoms, such as urinary retention and bradycardia, developed concurrently and persisted even after obtaining an improvement of the characteristic symptoms of PERM. Anti-gAChR $\alpha 3$ subunit antibodies are frequently identified in the serum of patients with autoimmune auto- 


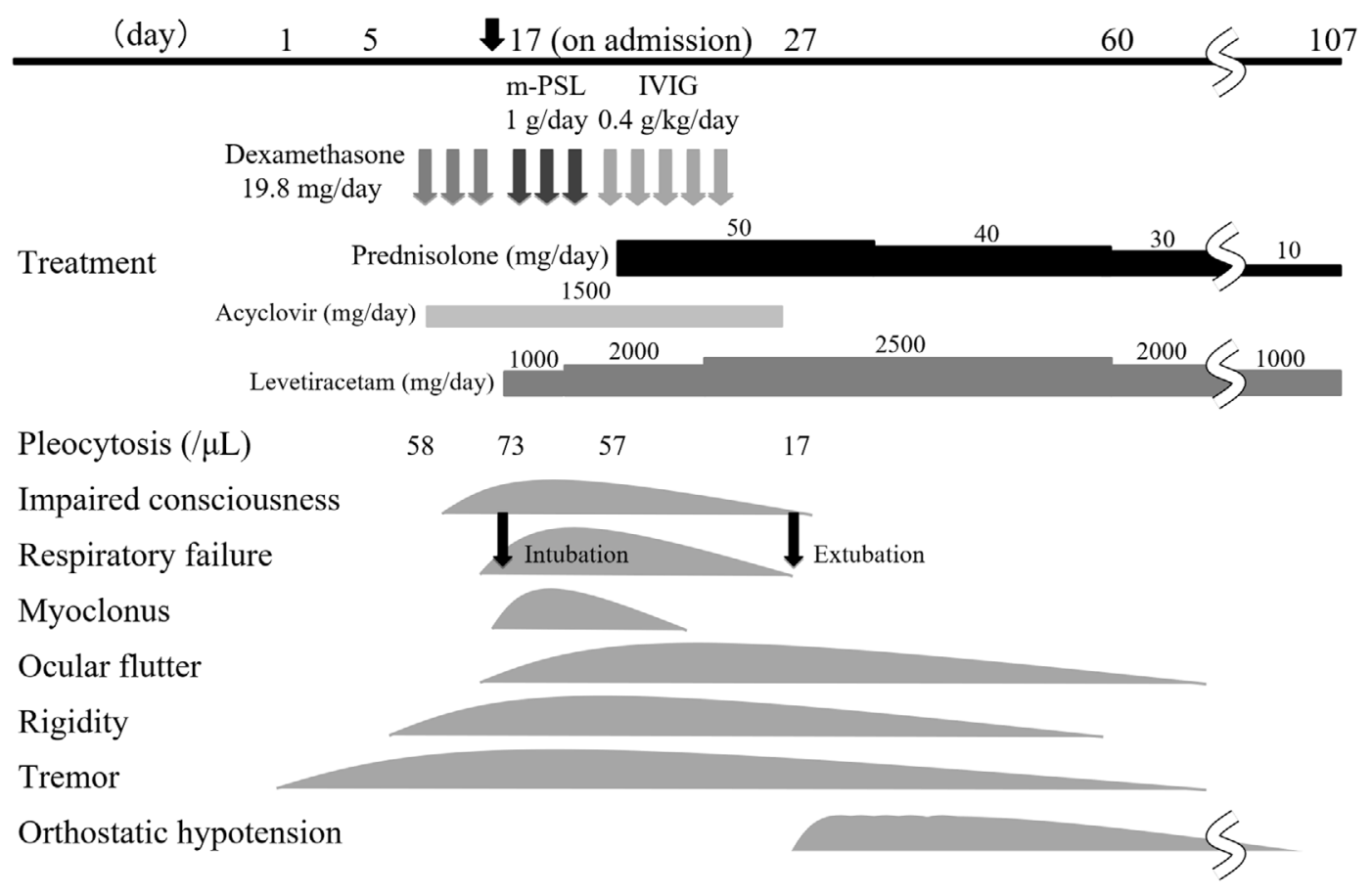

Figure 3. Clinical course of the present case. IVIG: intravenous immunoglobulin, m-PSL: methylprednisolone

nomic ganglionopathy (AAG) that presents with severe dysautonomia (8). Autonomic failure observed in AAG with anti-gAChR antibodies is mainly derived from the involvement of the non-sympathetic nervous system, including orthostatic hypotension in $83.3 \%$ (20/24 cases) and dysuria in $66.7 \%$ (16/24 cases) (8). These autonomic symptoms persist in most AAG cases (78\%: 62/80 cases) (17). Similar to our case, the reported cases of autoimmune encephalitis with anti-gAChR antibodies consistently presented with dysuria, orthostatic hypotension, and bradycardia $(18,19)$. Therefore, dysautonomia due to anti-AChR antibodies could be prolonged after immunotherapy.

On the other hand, 69\% (20/29 cases) of PERM with mainly anti-GAD antibodies and $43 \%$ of PERM with antiGlyR antibodies also presented with dysautonomia $(4,20)$. Stiff-person syndrome, including PERM, tends to show autonomic symptoms derived from the sympathetic nervous system, such as sweating, pupil dilation, tachycardia, tachypnea, hypertension, and hyperthermia (20). In contrast, our case's autonomic failure mainly consisted of nonsympathetic symptoms, such as orthostatic hypotension and bradycardia. The persistent and severe non-sympathetic autonomic failure in the present case provided us the momentum to measure anti-gAChR antibodies as the presence of other antibodies related to PERM including GAD, GlyR, NMDAR, and DPPX was negative. Although other mechanisms, including undetected autoantibodies, might have also been reported to be involved in the pathogenesis of PERMlike symptoms, our case's anti-gAChR antibodies were presumably associated with both PERM-like symptoms and non-sympathetic dysautonomia. Our case underlines the importance of measuring the anti-gAChR antibodies in patients with PERM-like features accompanied by prolonged nonsympathetic dysautonomia.

Considering the relationship between anti-gAChR $\alpha 3$ subunit antibodies and PERM-like manifestations, antigAChR $\alpha 3$ subunit antibodies mainly target the $\alpha 3$ subunit of neuronal $\alpha 3 \beta 4$-type nAChRs, which are highly expressed not only in the peripheral autonomic ganglia but also in the brainstem and spinal cord (7). Anti-gAChR antibodies reduce $\mathrm{nAChR}$ on the autonomic ganglion neurons in experimental animal models, resulting in autonomic failure (21). The IgG subclass of anti-gAChR antibodies has been reported as IgG3 in a recent study (22); however, further studies are necessary to confirm this subclass. Although the pathological effects of anti-gAChR antibodies on nAChR in the CNS remain unclear (23), suppression of nAChR functions in the CNS by anti-gAChR antibodies could result in PERM symptomatology: rigidity, myoclonus, seizures, and CNS lesions on MRI, as evidenced in the present case. This composition is similar to the pathogenesis of PERM associated with anti-GlyR antibodies, which are presumed to suppress GlyRs distributed mainly in the brainstem and spinal cord (4). A recently reported case of opsoclonus-myoclonus syndrome with anti-gAChR $\alpha 3$ subunit antibodies also developed ocular dyskinesia and myoclonus of the face and extremities (24). The aforementioned case report suggests that anti-gAChR $\alpha 3$ subunit antibodies are linked to several cholinergic nuclei within the brainstem, which participate in motor and ocular-motor pathways (24). In addition, the reversible brain MRI and SPECT abnormalities after immunotherapy, observed in reported cases of autoimmune encephalitis with anti-gAChR antibodies, suggest that anti-gAChR antibodies against nAChR in the CNS act functionally rather 
Table 1. The Clinical Findings of 45 Cases of Progressive Encephalomyelitis with Rigidity and Myoclonus (PERM) Associated with Anti-glycine Receptor Antibodies (4) and Comparisons with the Present Case.

\begin{tabular}{|c|c|c|}
\hline & (4) & $\begin{array}{l}\text { The present } \\
\text { case }\end{array}$ \\
\hline \multicolumn{3}{|l|}{ Clinical symptoms at peak } \\
\hline Spasms/stiffness/rigidity/myoclonus & $80 \%(24 / 30)$ & + \\
\hline Oculomotor disturbance & $53 \%(16 / 30)$ & + \\
\hline Trigeminal, facial and bulbar disturbance & $57 \%(17 / 30)$ & + \\
\hline Excessive startle & $57 \%(17 / 30)$ & - \\
\hline $\begin{array}{l}\text { Walking difficulties /falls, mostly } \\
\text { related to stiffness/rigidity/spasms }\end{array}$ & $80 \%(24 / 30)$ & + \\
\hline Limb paresis/pyramidal signs & $60 \%(18 / 30)$ & + \\
\hline Autonomic failure & $43 \%(13 / 30)$ & + \\
\hline $\begin{array}{l}\text { Cognitive impairment/encephalopathy/ } \\
\text { seizures }\end{array}$ & $50 \%(15 / 30)$ & + \\
\hline Sensory symptoms/pain & $47 \%(14 / 30)$ & + \\
\hline Respiratory failure & $27 \%(8 / 30)$ & + \\
\hline \multicolumn{3}{|l|}{ CSF findings } \\
\hline Pleocytosis & $43 \%(13 / 30)$ & + \\
\hline \multicolumn{3}{|l|}{ Brain MRI abnormalities } \\
\hline White matter lesions & $8 \%(3 / 36)$ & + \\
\hline Temporal lobe inflammation & $6 \%(2 / 36)$ & - \\
\hline Other FLAIR lesions & $6 \%(2 / 36)$ & $\begin{array}{c}+ \\
\text { (Brainstem } \\
\text { lesions) }\end{array}$ \\
\hline \multicolumn{3}{|l|}{ Spine MRI abnormalities } \\
\hline Short or patchy lesions & $17 \%(4 / 23)$ & + \\
\hline \multicolumn{3}{|l|}{ EEG abnormalities } \\
\hline Slow activity & $52 \%(11 / 21)$ & + \\
\hline Focal epileptic discharges & $14 \%(3 / 21)$ & - \\
\hline GlyR antibodies & $100 \%(45 / 45)$ & - \\
\hline \multicolumn{3}{|l|}{ Other autoantibodies } \\
\hline GAD antibodies & $9 \%(4 / 45)$ & - \\
\hline Thyroid antibodies & $21 \%(6 / 28)$ & + \\
\hline Tumors & $25 \%(5 / 20)$ & - \\
\hline
\end{tabular}

CSF: cerebrospinal fluid, EEG: electroencephalogram, FLAIR: fluid-attenuated inversion-recovery, GAD: glutamic acid decarboxylase, GlyR: glycine receptors, MRI: magnetic resonance imaging, PERM: progressive encephalomyelitis with rigidity and myoclonus

than destructively (19). Similarly, in our case, the CNS symptoms and abnormal brain-spinal MRI findings improved after immunotherapy; however, dysautonomia was refractory to immunotherapy. These findings suggest that anti-gAChR antibodies may militate reversibly against nAChR in the CNS while disrupting nAChR in the autonomic nervous system. Anti-gAChR antibodies may have simultaneously affected the CNS and peripheral autonomic ganglia in the early stages of the disease. However, in our case, the different behavior of anti-gAChR antibodies may have negatively influenced the recovery process.

Previous studies showed that CNS involvement, especially psychiatric symptoms, was observed in $31-45 \%$ of Japanese patients with AAG who were seropositive for anti-gAChR antibodies as assessed by LIPS assay method (the healthy control value was defined as A.I. <1.0) (17). However, there have been a few reports describing cases with anti-gAChR $\alpha$ 3 subunit antibodies that showed encephalitis with brain le- sions on MRI and pleocytosis in CSF $(18,19)$. These case reports showed that although the PERM features did not present, the presence of anti-gAChR $\alpha 3$ subunit antibodies and symptoms of autoimmune encephalitis improved after immunotherapy, similar to our case (Table 2). In these case reports, Baker et al. measured anti-gAChR $\alpha 3$ subunit antibody values using the radioimmunoprecipitation assay method, which showed $2.06 \mathrm{nmol} / \mathrm{L}$ (normal, <0.05) in their case (18). Kuki et al. measured the antibody index (A.I.) using the LIPS assay method, which exhibited 1.58 A.I. in their case (19). In addition to these case reports, we recently reported five cases of autoimmune encephalitis with antigAChR $\alpha 3$ subunit antibodies measured by the LIPS method (23). Although the present case has partially been reported in this study (23), the detailed clinical findings, especially PERM-like features, were not described. In the study of the five autoimmune encephalitis cases, the levels (i.e., A.I.) of anti-gAChR $\alpha 3$ subunit antibodies were 1.68土 
Table 2. Comparison of the Clinical Features of Past Cases That were Seropositive for Anti-ganglionic Acetylcholine Receptor Antibodies Presenting with Brain Lesions and Pleocytosis $(18,19)$ and the Present Case.

\begin{tabular}{|c|c|c|c|}
\hline & (18) & (19) & The present case \\
\hline Age, $(y) / \operatorname{Sex}$ & $47 / \mathrm{F}$ & $13 / \mathrm{M}$ & $59 / \mathrm{F}$ \\
\hline Diagnosis & $\mathrm{AE}$ & $\mathrm{AE}$ & PERM-like AE \\
\hline \multicolumn{4}{|l|}{ Clinical symptoms } \\
\hline Consciousness disturbance & + & + & + \\
\hline Respiratory failure & - & - & + \\
\hline Oculomotor disturbance & - & - & + \\
\hline Myoclonus & - & - & + \\
\hline Rigidity of limbs & - & - & + \\
\hline \multicolumn{4}{|l|}{ Autonomic failure } \\
\hline Orthostatic hypotension & + & + & + \\
\hline Sinus bradycardia & + & + & + \\
\hline Dysuria & + & + & + \\
\hline \multicolumn{4}{|l|}{ CSF findings } \\
\hline Pleocytosis & + & + & + \\
\hline Elevated protein & + & + & + \\
\hline \multicolumn{4}{|l|}{ MRI findings } \\
\hline Brain lesions & $\begin{array}{c}\text { Periventricular and } \\
\text { subcortical white matter }\end{array}$ & $\begin{array}{l}\text { Caudate nuclei, putamen, } \\
\text { hippocampus, and insula cortex }\end{array}$ & White matter and brainstem \\
\hline Spinal cord lesions & N/A & N/A & Cervical and thoracicthoracic \\
\hline \multicolumn{4}{|l|}{ NCS findings } \\
\hline CMAP and NCV & N/A & N/A & Normal \\
\hline F-wave disappearance & N/A & N/A & + \\
\hline \multicolumn{4}{|l|}{ Serum findings } \\
\hline Type of anti-gAchR antibody & $\alpha 3$ & $\alpha 3$ & $\alpha 3$ \\
\hline $\begin{array}{l}\text { Titer of anti-gAchR } \alpha 3 \text { antibody } \\
\text { (pre/post-treatment) }\end{array}$ & $\begin{array}{l}2.06 / 0.18 \mathrm{nmol} / \mathrm{L} \\
\quad(\text { normal },<0.05)\end{array}$ & $1.58 / 1.31$ A.I. (normal,<1) & 1.18 / 0.38 A.I. (normal, <1) \\
\hline Other positive antibodies & $\begin{array}{l}\text { Antibodies to CNS } \alpha 4 \\
\quad \text { and } \alpha 7 \mathrm{nAChRs}\end{array}$ & - & Antibodies to TPO and Tg \\
\hline Tumors & - & - & - \\
\hline Treatment & $\begin{array}{l}\text { m-PSL, IVIG, PE, } \\
\text { Azathioprine }\end{array}$ & m-PSL, IVIG & m-PSL, IVIG \\
\hline Treatment response & Partial & Partial & Partial \\
\hline
\end{tabular}

AE: autoimmune encephalitis, A.I.: antibody index, anti-gAChR: anti-ganglionic acetylcholine receptor, CNS: central nervous system, CSF: cerebrospinal fluid, EEG: electroencephalogram, IVIG: intravenous immunoglobulin, N/A: not available, m-PSL: methylprednisolone, MRI: magnetic resonance imaging, NCS: nerve conduction study, PE: plasma exchange, PERM: progressive encephalomyelitis with rigidity and myoclonus, TPO: thyroid peroxidase, Tg: thyroglobulin

0.49 (mean \pm standard deviation) (23), which is similar to those in patients with AAG who were positive for antigAChR $\alpha 3$ subunit antibodies (1.529 \pm 0.634$)$ (25). Although the present case showed relatively lower A.I. in the pretreatment serum (1.18), the level was above the cutoff value $(<1.0)$ and it decreased after immunotherapy (0.568). Since a measurement of anti-AChR antibodies in CSF has not been validated, an evaluation of CSF was not performed in our case.

In the present case, PERM-like symptoms of both CNS manifestations and autonomic failure eventually improved with the negative conversion of anti-gAChR $\alpha 3$ subunit antibodies after immunotherapy, confirming the immunopathogenesis of anti-gAChR $\alpha 3$ subunit antibodies. The aforementioned two reported cases of autoimmune encephalopathy associated with anti-gAChR $\alpha 3$ subunit antibodies also showed a reduction of anti-gAChR $\alpha 3$ subunit antibody titer after immunotherapy $(18,19)$ (Table 2). These reported cases showed improved clinical symptoms of both CNS manifestations and autonomic failure along with the decreased titers, which was similar to our case.

The present case showed positive results of both anti-TPO and $\mathrm{Tg}$ antibodies as well as anti-gAChR $\alpha 3$ subunit antibodies, which suggests Hashimoto encephalopathy as a differential diagnosis (26). Impaired consciousness (66\%), convulsions (29\%), and involuntary movements including tremor and myoclonus (31\%), which were seen in our case, are often observed in patients with Hashimoto encephalopathy (26). However, the other symptoms seen in our case, especially autonomic failure and myelopathy, are uncommon in Hashimoto encephalopathy (26). In addition, immunological studies yielded negative anti-NAE antibodies, which are highly specific to Hashimoto encephalopathy (27), also suggesting a low probability of Hashimoto encephalopathy in our case.

We herein described a case of PERM-like symptoms asso- 
ciated with anti-gAChR antibodies. The distribution of the target nAChRs in the CNS and peripheral autonomic nerves explains the PERM-like symptoms and autonomic failure. Thus, the clinical spectrum associated with anti-gAChR antibodies is thus considered to extend from AAG to PERM.

The authors state that they have no Conflict of Interest (COI).

\section{Acknowledgement}

The authors thank Prof. Makoto Yoneda (Department of Nursing Science, Graduate School of Nursing and Social Welfare Sciences, Fukui Prefectural University) and Prof. Josep Dalmau (Institucio Catalana de Recerca i Estudis Avancats (ICREA) at Institut d'Investigacio Biomedica August Pi i Sunyer (IDIBAPS), Service of Neurology, Hospital Clinic, University of Barcelona, Department of Neurology, University of Pennsylvania) for their help with screening the serum for autoimmune encephalitisrelated autoantibodies.

\section{References}

1. Whiteley AM, Swash M, Urich H. Progressive encephalomyelitis with rigidity. Brain 99: 27-42, 1976.

2. Thompson PD. The stiff-man syndrome and related disorders. Parkinsonism Relat Disord 8: 147-153, 2001.

3. Barker RA, Revesz T, Thom M, Marsden CD, Brown P. Review of 23 patients affected by the stiff man syndrome: clinical subdivision into stiff trunk (man) syndrome, stiff limb syndrome, and progressive encephalomyelitis with rigidity. J Neurol Neurosurg Psychiatry 65: 633-640, 1998.

4. Carvajal-González A, Leite MI, Waters P, et al. Glycine receptor antibodies in PERM and related syndromes: characteristics, clinical features and outcomes. Brain 137: 2178-2192, 2014.

5. Turner MR, Irani SR, Leite MI, Nithi K, Vincent A, Ansorge O. Progressive encephalomyelitis with rigidity and myoclonus: glycine and NMDA receptor antibodies. Neurology 77: 439-443, 2011.

6. Balint B, Jarius S, Nagel S, et al. Progressive encephalomyelitis with rigidity and myoclonus: a new variant with DPPX antibodies. Neurology 82: 1521-1528, 2014.

7. Dineley KT, Pandya AA, Yakel JL. Nicotinic ACh receptors as therapeutic targets in CNS disorders. Trends Pharmacol Sci 36: 96-108, 2015.

8. Nakane S, Higuchi O, Koga M, et al. Clinical features of autoimmune autonomic ganglionopathy and the detection of subunitspecific autoantibodies to the ganglionic acetylcholine receptor in Japanese patients. PLOS ONE 10: e0118312, 2015.

9. Meinck HM, Thompson PD. Stiff man syndrome and related conditions. Mov Disord 17: 853-866, 2002.

10. Ueno S, Miyamoto N, Shimura H, et al. Successful immune moderation treatment for progressive encephalomyelitis with rigidity and myoclonus. Intern Med 54: 219-221, 2015.

11. Peeters E, Vanacker P, Woodhall M, et al. Supranuclear gaze palsy in glycine receptor antibody-positive progressive encephalomyelitis with rigidity and myoclonus. Mov Disord 27: 1830-1832, 2012.

12. Kraemer M, Berlit P. Progressive encephalomyelitis with rigidity and myoclonus in an 81-year-old patient. Clin Neurol Neurosurg 110: 279-281, 2008.

13. Xu Z, Prasad K, Yeo T. Progressive encephalomyelitis with rigidity and myoclonus in an intellectually disabled patient mimicking neuroleptic malignant syndrome. J Mov Disord 10: 99-101, 2017.

14. Degeneffe A, Dagonnier M, D'hondt A, Elosegi JA. A case report of rigidity and recurrent lower limb myoclonus: progressive encephalomyelitis rigidity and myoclonus syndrome, a chameleon. BMC Neurol 18: 173, 2018.

15. Dalakas MC, Fujii M, Li M, McElroy B. The clinical spectrum of anti-GAD antibody-positive patients with stiff-person syndrome. Neurology 55: 1531-1535, 2000.

16. Strawn JR, Keck PE Jr, Caroff SN. Neuroleptic malignant syndrome. Am J Psychiatry 164: 870-876, 2007.

17. Nakane S, Mukaino A, Maeda Y, Higuchi H, Matsuo H, Ando Y. Extra-autonomic manifestations in autoimmune autonomic ganglionopathy: a Japanese survey. J Neurol Neurosurg Psychiatry 88: 367-368, 2017.

18. Baker SK, Morillo C, Vernino S. Autoimmune autonomic ganglionopathy with late-onset encephalopathy. Auton Neurosci 146: 29-32, 2009.

19. Kuki I, Kawawaki H, Okazaki S, et al. Autoimmune autonomic ganglionopathy in a pediatric patient presenting with acute encephalitis. Brain Dev 38: 605-608, 2016.

20. Meinck HM, Thompson PD. Stiff man syndrome and related conditions. Mov Disord 17: 853-866, 2002.

21. Vernino S, Low PA, Lennon VA. Experimental autoimmune autonomic neuropathy. J Neurophysiol 90: 2053-2059, 2003.

22. Kobayashi S, Yokoyama S, Maruta T, et al. Autoantibody-induced internalization of nicotinic acetylcholine receptor $\alpha 3$ subunit exogenously expressed in human embryonic kidney cells. J Neuroimmunol 257: 102-106, 2013.

23. Yamakawa M, Mukaino A, Kimura A, et al. Antibodies to the $\alpha 3$ subunit of the ganglionic-type nicotinic acetylcholine receptors in patients with autoimmune encephalitis. J Neuroimmunol 349: 577399, 2020.

24. Galli JR, Clardy SL, Paz Soldán MM. Adult-onset opsoclonusmyoclonus syndrome associated with ganglionic acetylcholine receptor autoantibody. Neurologist 21: 99-100, 2016.

25. Nakane S, Mukaino A, Higuchi O, et al. A comprehensive analysis of the clinical characteristics and laboratory features in 179 patients with autoimmune autonomic ganglionopathy. J Autoimmun 108: 102403, 2020.

26. Matsunaga A, Ikawa M, Yoneda M. Hashimoto encephalopathy. Clin Exp Neuroimmunol 10: 226-233, 2019.

27. Yoneda M, Fujii A, Ito A, et al. High prevalence of serum autoantibodies against the amino terminal of $\alpha$-enolase in Hashimoto's encephalopathy. J Neuroimmunol 185: 195-200, 2007.

The Internal Medicine is an Open Access journal distributed under the Creative Commons Attribution-NonCommercial-NoDerivatives 4.0 International License. To view the details of this license, please visit (https://creativecommons.org/licenses/ by-nc-nd/4.0/). 